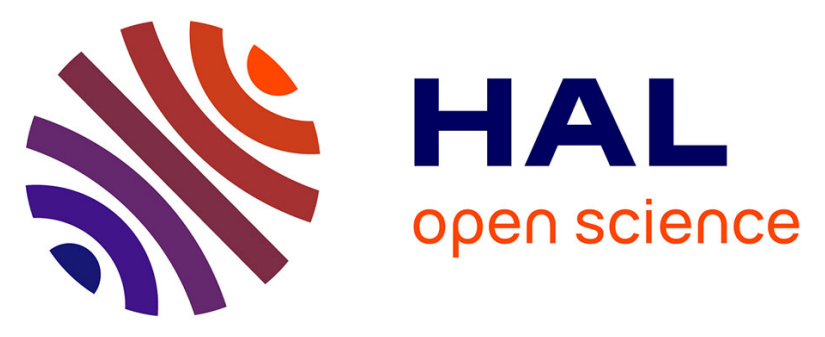

\title{
Upscaling electrokinetic transport in clays with lattice Boltzmann and Pore Network Models
}

\author{
Amaël Obliger, Marie Jardat, Daniel Coelho, Samir Bekri, Benjamin
}

Rotenberg

\section{- To cite this version:}

Amaël Obliger, Marie Jardat, Daniel Coelho, Samir Bekri, Benjamin Rotenberg. Upscaling electrokinetic transport in clays with lattice Boltzmann and Pore Network Models. The Clay Minerals Society. CMS Workshop Lectures, Volume 21: Filling The Gaps-From Microscopic Pore Structures To Transport Properties In Shales, 21, , pp.129-135, 2016, Clay Minerals Society Workshop Lectures Series, 10.1346/CMS-WLS-21.10 . hal-01484797

\section{HAL Id: hal-01484797 \\ https://hal.sorbonne-universite.fr/hal-01484797}

Submitted on 8 Nov 2018

HAL is a multi-disciplinary open access archive for the deposit and dissemination of scientific research documents, whether they are published or not. The documents may come from teaching and research institutions in France or abroad, or from public or private research centers.
L'archive ouverte pluridisciplinaire HAL, est destinée au dépôt et à la diffusion de documents scientifiques de niveau recherche, publiés ou non, émanant des établissements d'enseignement et de recherche français ou étrangers, des laboratoires publics ou privés. 


\section{UPSCALING ELECTROKINETIC TRANSPORT IN CLAYS} WITH LATTICE BOLTZMANN AND PORE NETWORK MODELS

SHORT TITLE: Upscaling electrokinetic transport in clays

Corresponding author: Benjamin Rotenberg (benjamin.rotenberg@upmc.fr), UMR PHENIX, UPMC, 4 place Jussieu, 75005 Paris France

\section{ABSTRACT}

This contribution presents a method for the numerical determination of the steady-state response of complex charged porous media to pressure, salt concentration and electric potential gradients. The Pore Network Model (PNM), describing the porosity as a network of pores connected by channels, is extended to capture electrokinetic couplings which arise at charged solid-liquid interfaces. This allows us to compute the macroscopic fluxes of solvent, salt and charge across a numerical sample submitted to macroscopic gradients. On the channel scale, the microscopic transport coefficients are obtained by solving analytically (in simple cases) or numerically the Poisson-Nernst-Planck and Stokes equations. The PNM approach then allows us to upscale these transport properties to the sample scale, accounting for the complex pore structure of the material via the distribution of channel diameters. The Onsager relations between macroscopic transport coefficients are preserved, as expected. However, electrokinetic couplings combined with the sample heterogeneity result for some macroscopic transport coefficients (e.g. permeability or electro-osmotic coefficient) in qualitative differences with respect to their microscopic counterparts. This underlines the care that should be taken when accounting for transport properties based on a single channel of average diameter.

32 KEYWORDS: Electrokinetics, Coupled transport, Homogenization, Lattice Boltzmann, Pore

33 Network Model, Upscaling 
36 Electrokinetic effects refer to the dynamic coupling between the solvent and charge flows which 37 occur at a charged interface. The presence of surface charge in a porous medium has important 38 practical applications in membrane technology (e.g., ion exchange and water desalination) and in environmental science, since most rocks and soils contain minerals (such as clays) that bear a permanent surface charge. As an example, electro-osmosis generates a solvent flow under an applied electric field, due to the driving of the electrically charged fluid in the vicinity of charged surfaces. Conversely, a pressure gradient induces the flow of a charged fluid, hence, an electric current. In geophysics, the electroseismic effect, by which an electro-magnetic wave is generated from the motion of underground fluids under an applied acoustic wave, is exploited to determine the properties of geological formations (Thompson, 1936; Pride and Haartsen, 1996; Mizutani et al., 1976). Streaming potentials and electro-osmotic flows can be measured in the laboratory to characterize the properties of porous media (Luong and Sprik, 2013).

The modeling and simulation of electrokinetic effects in porous media, and, more generally, of all coupled transport phenomena, including the osmotic solvent flow due to a salt concentration gradient, thus have been the subject of a large number of investigations, both on the pore scale where the couplings originate and on the sample scale corresponding to the experimental measurements. From the mathematical point of view, this upscaling can be performed rigorously using the homogenization approach. This provides expressions of the macroscopic transport coefficients as solutions of coupled partial differential equations on the pore scale, which then have to be solved using simplifying assumptions or numerically. Some general results, such as Onsager's relations for the macroscopic transport coefficients, can be demonstrated without even resorting to the numerical resolution of the mathematical problem (Moyne and Murad, 2006a\&b; Allaire et al., 2010\&2014).

For practical applications, most studies of electrokinetic couplings rely on an oversimplified idealization of the geometry, with single slit pores or cylinders with dimensions or surface

63 charge densities estimated from the macroscopic properties of the real system (Bresler, 1973; 64 Gonçalvès et al., 2012). However, the heterogeneity of the material, combined with the 
65 electrokinetic couplings, may influence the overall behavior on the sample scale, so that such 66 idealizations may not reflect the actual response of the medium. Direct numerical resolution of 67 the coupled Poisson-Nernst-Planck (PNP) and Navier-Stokes (NS) equations in various complex 68 systems (random packings, reconstructed and fractured porous media) has also been proposed by 69 Adler and co-workers. Such an approach is usually difficult to implement for macroscopic samples, due to the lack of experimental data on the fine structure of the material over large

71 distances (Coelho et al., 1996; Marino et al., 2001; Gupta et al., 2006). The systematic study of a 72 representative number of samples is also prevented by the computational cost of direct numerical 73 simulation.

In the case of clays, an additional difficulty arises due to the complex multiscale porosity of the material and the lack of experimental data on the intermediate scales, which is at the heart of this workshop. In the present contribution, we present a numerical homogenization scheme leading to a description of transport through macroscopic charged porous materials at low computational cost, thereby enabling the systematic study of the combined effects of electrokinetic couplings and sample heterogeneity. The algorithm to upscale the electrokinetic couplings is based on the

81 Pore Network Model (PNM), which relies on the one hand on a simplified description of the 82 electrokinetic transport on the pore scale and on the other hand on a statistical distribution of the geometry of the pores. This allows to investigate how the upscaled electrokinetic properties

84 depend on the heterogeneity of the sample, in addition to the surface charge density and the salt 85 concentration.

\section{ELECTROKINETICS ON THE SAMPLE SCALE}

On the macroscopic scale of a clay sample, pressure $\mathrm{P}$, electric potential V and salt concentration gradients (or, equivalently, solvent, cation and anion chemical potentials gradients), induce macroscopic fluxes of mass, electric charge and salt (or, equivalently, solvent, cation and anion

93 chemical fluxes). For sufficiently small applied gradients, the response is linear and the fluxes 94 can be expressed as a function of the applied gradients via a coupling matrix: 


$$
\left(\begin{array}{c}
\mathcal{Q}_{0} \\
\mathcal{Q}_{1} \\
\mathcal{Q}_{2}
\end{array}\right)=-\frac{S}{\eta}\left(\begin{array}{ccc}
K_{0}^{P} & K_{0}^{C} & K_{0}^{V} \\
K_{1}^{P} & K_{1}^{C} & K_{1}^{V} \\
K_{2}^{P} & K_{2}^{C} & K_{2}^{V}
\end{array}\right)\left(\begin{array}{c}
\nabla P \\
\nabla C \\
\nabla V
\end{array}\right),
$$

where the subscripts 0,1 and 2 refer to solvent, cations and anions, respectively, $Q$ indicate their

fluxes, $\mathrm{C}$ is the logarithm of the salt concentration, $\eta$ the solvent viscosity and $\mathrm{S}$ the cross-section

100 of the sample. Along the diagonal one finds for example the sample permeability $\mathrm{K}_{0}{ }^{\mathrm{P}}$ and the 101 electric conductivity $\mathrm{K}_{2} \mathrm{~V}$. The fundamental question is then: How do these coefficients emerge

102 from the microscopic structure of the material, including heterogeneities on intermediate scales, 103 and from the surface charge density of the solid matrix?

\section{ELECTROKINETICS ON THE PORE SCALE}

Recently, significant progress has been made on the derivation of the macroscopic transport equations from the pore-scale ones. These studies usually start from a continuous description of the fluid via transport equations, which are then upscaled to derive their average effect on the sample scale, which is quantified by a coupling matrix relating the solvent and ionic fluxes to the corresponding forces (pressure, potential, and concentration gradients). The solvent flow under applied local forces is accounted for via the NS equation (or even the Stokes equation), which includes a local force due to electrochemical potential gradients. The solute fluxes are due one the one hand to the advection by the fluid and on the other hand to the local electrochemical potential gradients; they can be modelled on this scale using the PNP equations. The limitations of such continuous descriptions to describe solvent and ion transport in clay nanopores, which can be assessed using molecular simulations (Botan et al., 2010\&2013), will not be discussed here. Rather, the present discussion focusses on how to upscale this to the macroscopic scale,

122 The coupled Navier-Stokes and Poisson-Nernst-Planck equations can be solved numerically 123 using finite element or volume methods. For example, Adler and co-workers used this direct 124 numerical resolution in various complex systems (random packings, reconstructed and fractured 125 porous media) (Coelho et al., 1996; Marino et al., 2001), demonstrating in particular a universal 126 electrokinetic behaviour if appropriate rescaled quantities are introduced (Gupta et al., 2006, 
127 2008). Recently, alternative methods have been proposed to simulate electrokinetic effects 128 starting from a more fundamental description of the fluid than the PNP and NS equation 129 (Pagonabarraga et al., 2010). For example, Capuani et al. proposed a hybrid lattice based 130 approach (Lattice Boltzmann Electrokinetics, LBE) to capture the coupling of hydrodynamic 131 flow with ion transport and the simulation of electrokinetic effects in colloidal suspensions 132 (Capuani et al., 2004; Pagonabarraga et al., 2005). Such Lattice Boltzmann simulations have 133 already been applied, without accounting for electrokinetic effects, to realistic rock geometries 134 (Boek and Venturoli, 2010). In the context of the present numerical homogenization, LBE was 135 recently used in a simple cylindrical geometry, in order to assess the validity range of the 136 analytical solution of the linearized problem (Obliger et al., 2013). This simpler analytical 137 solution is then used in the PNM, even though in principle a numerical expression for the transport coefficient on the pore scale may also be used.

\section{NUMERICAL HOMOGENIZATION VIA A PORE NETWORK MODEL}

143 In order to investigate electrokinetic couplings on larger scales, including the effect of the 144 heterogeneity of the material, we have recently proposed a simplified description based on the 145 Pore Network Model (PNM). Such a model, originally developed by Fatt (1956) to predict 146 multiphase flow properties in porous media, describes the porosity as a network of pores 147 connected by channels. It has been extensively used and extended by petrophysicists in various 148 situations, such as capillarity and multiphase flow through porous media (Békri et al., 2005; 149 Blunt, 2001; van Dijke and Sorbie, 2002), or mineral dissolution and precipitation in the context 150 of $\mathrm{CO}_{2}$ sequestration (Algive et al., 2010).

152 In a nutshell, the PNM approach amounts to solving a set of conservation equations on the nodes 153 of the network (in analogy with Kirchhoff's law for a network of resistors), on the basis of local 154 fluxes through the channels connecting the nodes, under the effect of an external, macroscopic 155 gradient. For electrokinetics, the pressure, salt concentration and electrical potential are 156 introduced as pore variables on the nodes of the network. The fluxes through each link between 157 nodes are determined locally using the transport matrix for a cylindrical channel, as determined 
158 in the previous section as a function of the channel diameter, the surface charge density of the 159 solid and the salt concentration inside the channel. The latter is determined via the Donnan 160 equilibrium with a fictitious reservoir corresponding to the properties of the pores at both ends of 161 the channel (Obliger et al., 2014). Therefore, the macroscopic problem to be solved numerically 162 has a non-linear structure, contrary to most previous applications of the PNM approach. This can 163 be achieved numerically using a non-linear Newton solver.

TRANSPORT COEFFICIENTS ON THE SAMPLE SCALE

In addition to the transport coefficients on the channel scale, the crucial ingredient of the PNM is the distribution of pore/channel sizes and their spatial arrangement describing in a very simplified manner the complex structure of the porous network. In order to demonstrate the

171 feasibility of the approach and to investigate systematically the effect of heterogeneity, a model 172 distribution (of the Weibull type) was considered first. However, it is also possible to introduce a 173 distribution deduced from experimental data, if a reliable one can be provided.

175 For a given pore/channel diameter distribution, a sufficient number of networks must be generated. For each of numerical sample, the macroscopic coefficients are determined by solving

177 the conservation equations in the presence of applied gradients and by computing the 178 macroscopic steady-state flux through the sample. In practice, three calculations must be done 179 (one for each applied gradient) for which the three fluxes (mass, charge, salt concentration) are 180 computed. This provides the nine macroscopic coefficients, which must then be averaged over 181 the networks corresponding to the same diameter distribution. This general approach will be 182 illustrated during the workshop on a number of test cases.

\section{CONCLUSION}

During the workshop, the various steps of the proposed PNM approach will be presented and its interest illustrated for charged porous materials. The influence of the surface charge density, the 
190 salt concentration in the reservoirs and of the channel diameter distribution will be analyzed. The 191 symmetry of the transport matrix is preserved by the present upscaling method, as required from 192 Onsager's theory. In general, the coefficients of this matrix qualitatively behave as their 193 microscopic counterpart for a channel with the average diameter. However, the combined effects 194 of electrokinetic couplings on the local scale and of heterogeneity result in a decrease of the 195 overall transport coefficients, in accordance with Le Châtelier's principle. Overall, the coupling 196 between the complex pore structure of porous media and electrokinetic effects underlines the

197 limitations of approaches based on idealized geometries (single slit pore or cylindrical channel) 198 parametrized directly from the experimental macroscopic properties.

200 The relevance and limitations of this new strategy to the case of clay minerals will be discussed. 201 In that respect, experimental information on the pore network and its size distribution on 202 intermediate $(10-100 \mathrm{~nm})$ scales is highly desirable for the present method to provide more 203 quantitative predictions in this case. In the future, one should benefit from recent numerical 204 (Tyagi et al., 2013) and experimental (Brisard et al., 2012; Levitz, 2007) developments for the 205 generation of realistic numerical samples for the description of real materials. As a recent 206 example, Robinet et al. recently simulated the diffusion of solutes in 3D-images of a Callovo207 Oxfordian clay-rich rock obtained by SEM and micro-CT experiments to investigate the effect of 208 mineral distribution (Robinet et al., 2012). Multiscale experiments using NMR also provide an 209 ideal tool to investigate themultiscale dynamics of mobile species in such complex materials 210 (Porion et al., 2013). 


\section{REFERENCES}

Algive, L., Békri, S. and Vizika, O. (2010). Pore-network modeling dedicated to the determination of the petrophysical-property changes in the presence of reactive fluid. $S P E$ Journal 15, 124305.

Allaire, G., Mikelic, A. and Piatnitski, A. (2010). Homogenization of the linear- ized ionic transport equations in rigid periodic porous media. Journal of Mathematical Physics 51, 123103.

Allaire, G., Brizzi, R., Dufrêche, J.F., Mikelic, A. and Piatnitski, A. (2014). Role of non-ideality for the ion transport in porous media: derivation of the macroscopic equations using upscaling. Physica D 282, 39.

Békri, S., Laroche, C. and Vizika, O. (2005). Pore network models to calculate transport and electrical properties of single or dual-porosity rocks. Abstracts of the Society of Core Analysts, 35 .

Blunt, M.J. (2001). Flow in porous media pore-network models and multi-phase flow. Current Opinions in Colloid and Interface Science 6, 197-207.

Boek, E.S. and Venturoli, M. (2010). Lattice-Boltzmann studies of fluid flow in porous media with realistic rock geometries. Computers \& Mathematics with Applications 59, 2305-2314.

Botan, A., Rotenberg, B., Marry, V., Turq, P. and Noetinger, B. (2011). Hydrodynamics in clay nanopores. The Journal of Physical Chemistry C 115, 16109-16115.

Botan, A., Marry, V., Rotenberg, B., Turq, P. and Noetinger, B. (2013). How electrostatics influences hydrodynamic boundary conditions: Poi- seuille and electro-osmostic flows in clay nanopores. The Journal of Physical Chemistry C 117, 978-985.

Bresler, E. (2010). Simultaneous transport of solutes and water under transient unsaturated flow conditions. Water Resources Research 9, 975.

Brisard, S., Chae, R.S., Bihannic, I., Michot, L., Guttmann, P., Thieme, J., Schneider, G., Monteiro, P.J.M. and Levitz, P. (2012). Morphological quantification of hierarchical geomaterials by X-ray nano-CT bridges the gap from nano to micro length scales. American Mineralogist 97, 480-483.

Coelho, D., Shapiro, M., Thovert, J.F. and Adler, P.M. (1996). Electroosmotic phenomena in porous media. J Journal of Colloid and Interface Science 181, 169-190.

van Dijke, M.I.J. and Sorbie, K.S. (2002). Pore-scale network model for three-phase flow in mixed-wet porous media. Physical Review E 66, 046302. 
Fatt, I. (1956). The network model of porous media. Part I. Capillary characteristics. Petroleum Transactions AIME 207, 144-159.

Gonçalves, J., de Marsily, G. and Tremosa, J. (2012). Importance of thermo-osmosis for fluid Science Letters 339, 1.

Gupta, A.K., Coelho, D. and Adler, P.M. (2006). Electroosmosis in porous solids for high zeta potentials. Journal of Colloid and Interface Science 303, 593-603.

Gupta, A., Coelho, D. and Adler, P. (2008). Universal electro-osmosis formulae for porous media. Journal of Colloid and Interface Science 319, 549-554.

Levitz, P. (2007). Toolbox for 3D imaging and modeling of porous media: relationship with transport properties. Cement and Concrete Research 37, 351- 359.

Luong, D.T. and Sprik, R. (2013). Streaming Potential and Electroosmosis Measurements to Characterize Porous Materials. ISNR Geophysics 1, 496352.

Marino, S., Shapiro, M. and Adler, P. (2001). Coupled transports in heterogeneous media. Journal of Colloid and Interface Science 243, 391-419.

Mizutani, H., Ishido, T., Yokokura, T. and Ohnishi, S. (1976). Electrokinetic phenomena associated with earthquakes, Geophysical Research Letters 3, 365.

Moyne, C. and Murad, M.A., (2006a). A two-scale model for coupled electro-chemo-mechanical phenomena and Onsager's reciprocity relations in expansive clays. I. Homogenization analysis. Transport in Porous Media 62, 333-380.

Moyne, C. and Murad, M.A. (2006b). A two-scale model for coupled electro-chemo-mechanical phenomena and Onsager's reciprocity relations in expansive clays. II. Computational validation. Transport in Porous Media 63, 13-56.

Obliger, A., Duvail, M., Jardat, M., Coelho, D., Békri, S. and Rotenberg, B. (2013). Numerical homogenization of electrokinetic equations in porous media using lattice-Boltzmann simulations. Physical Review E 88, 013019.

Obliger, A., Jardat, M., Coelho, D., Békri, S. and Rotenberg, B. (2014). Pore network model of electrokinetic transport through charged porous media. Physical Review E 89, 043013.

Pagonabarraga, I., Capuani, F. and Frenkel, D. (2005). Mesoscopic lattice modeling of electrokinetic phenomena. Computer Physics Communication 169, 192-196.

Pagonabarraga, I., Rotenberg, B. and Frenkel, D. (2010). Recent advances in the modelling and simulation of electrokinetic effects: bridging the gap between atomistic and macroscopic descriptions. Physical Chemistry Chemical Physics 12, 9566-9580. 
307 Porion, P., Faugère, A.M. and Delville, A. (2013). Multiscale water dynamics within dense clay 308 sediments probed by ${ }^{2} \mathrm{H}$ multiquantum NMR relaxometry and two-time stimulated echo NMR

Pride, S.R. and Haartsen, M.W. (1996). Electroseismic wave properties. Journal of the Acoustic Society of America 100, 1301.

Robinet, J.C., Sardini, P., Coelho, D., Parneix, J.C., Prêt, D., Sammartino, S., Boller, E. and Altmann, S. (2012). Effects of mineral distribution at meso- scopic scale on solute diffusion in a clay-rich rock: example of the Callovo-Oxfordian mudstone (Bure, France). Water Resources Research 48, W05554.

Thompson, R.R. (1936). The seismic electric effect, Geophysics 1, 327.

Tyagi, M., Gimmi, T. and Churakov, S.V. (2013). Multi-scale micro-structure generation strategy for up-scaling transport in clays. Advances in Water Resources 59, 181-195. 